\title{
Evaluation of the advanced artificial athlete and Hall effect sensors for measuring strain in multi-layer sports surfaces
}

\author{
David Cole $^{1} \cdot$ Paul Fleming $^{2} \cdot$ Kelly Morrison $^{3} \cdot$ Steph Forrester $^{1}$
}

Received: 18 April 2019 / Accepted: 24 January 2020 / Published online: 24 February 2020

(C) The Author(s) $2020 \quad$ OPEN

\begin{abstract}
Computer models are a useful means to explore the loading behaviour of third generation (3G) artificial turf sports surfaces; however, measuring the material stress-strain behaviour under realistic high loading rates is challenging. Therefore, the purpose of this study was two-fold: to evaluate the advanced artificial athlete (AAA) for measuring strain behaviour of polymeric sports surfaces under high loading rates typical of player interactions; and to evaluate Hall effect sensors (HES) for measuring strain behaviour of an individual layer within multi-layer sports surfaces. An independent optical measurement system (GOM) provided gold standard sample deformation and strain. Forty-five impacts onto three test samples were measured simultaneously using the three systems. Poor agreement was found between AAA and GOM peak sample deformations and strain (systematic bias $2.4 \mathrm{~mm}, 95 \%$ confidence intervals $\pm 1.3 \mathrm{~mm}$, strain RMSD 29\%), largely attributable to errors in the AAA time of initial contact. Using a regression equation to correct this time led to much better agreement in AAA peak deformations and strain (systematic bias $0.1 \mathrm{~mm}, 95 \%$ confidence intervals $\pm 0.7 \mathrm{~mm}$, strain RMSD 8\%). Good agreement was found between the HES and GOM (systematic bias $0.2 \mathrm{~mm}, 95 \%$ confidence intervals $\pm 0.4 \mathrm{~mm}$, strain RMSD 11\%). The corrected AAA and HES methods can measure deformation of polymeric sports surfaces under realistic loading rates to an accuracy of $<1 \mathrm{~mm}$. In terms of strain, errors increase with decreasing peak sample deformation indicating that both systems should be used with caution for peak deformations $<\sim 4-5 \mathrm{~mm}$.
\end{abstract}

Keywords Artificial turf $\cdot$ Advanced artificial athlete $\cdot$ Surface deformation $\cdot$ Hall effect sensors

$\begin{array}{ll}\text { Abbreviations } \\ \text { 3G } & \text { Third generation } \\ \text { AAA } & \text { Advanced artificial athlete } \\ a_{p k} & \text { Peak acceleration } \\ \text { FIFA } & \text { Fédération Internationale de Football } \\ & \text { Association } \\ \text { HES } & \text { Hall effect sensors } \\ \text { RMSD } & \text { Root mean square deviation } \\ \text { RS } & \text { BSW Berleburger Regupol Kombi } 1100 \\ \text { SP1 } & \text { BSW Berleburger Regupol 6010 SP } \\ \text { SP2 } & \text { Recticel Re-bounce F 82.01 } \\ T 1 & \text { Time of peak impact velocity } \\ \text { T2 } & \text { Time of surface contact }\end{array}$

\section{Introduction}

Third generation (3G) artificial turf is a common alternative to natural turf for sports pitches in climates where extreme weather, such as freezing temperatures or lack or water, can make it difficult to maintain a high quality natural turf [1]. Third generation artificial turf is constructed from a number of layers with a range of materials and properties (Fig. 1) [2, 3]. The bottom layer consists of an elastomeric shockpad designed to absorb impacts. On top of this lies the carpet, consisting of tufts of polymer fibres stitched into a canvas backing. The carpet layer also contains two infills: at the base is

\footnotetext{
$\triangle$ Steph Forrester, s.forrester@lboro.ac.uk| ${ }^{1}$ Sports Technology Institute, Wolfson School of Mechanical, Electrical and Manufacturing Engineering, Loughborough University, Loughborough LE11 3TU, UK. ${ }^{2}$ School of Architecture, Building and Civil Engineering, Loughborough University, Loughborough LE11 3TU, UK. ${ }^{3}$ Department of Physics, Loughborough University, Loughborough LE11 3TU, UK.
} 


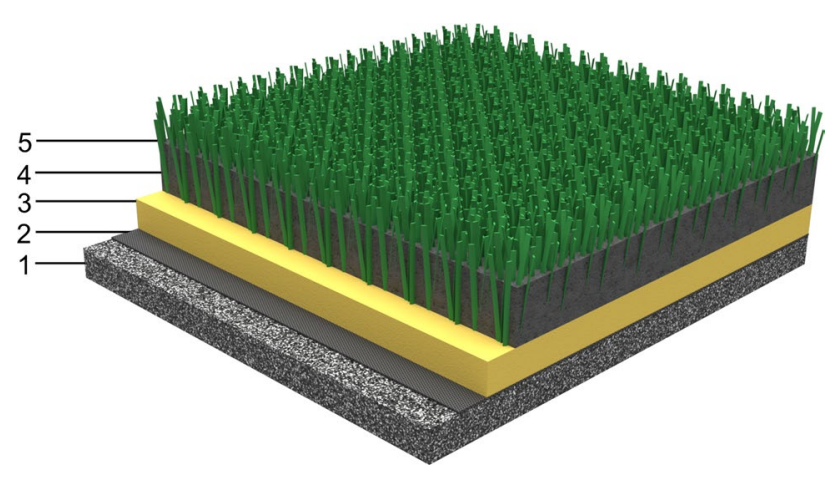

Fig. 1 Layered construction of a third generation (3G) turf surface: 1. Shockpad; 2. Carpet backing; 3. Stabilising infill; 4. Performance infill; 5. Fibres

a stabilising infill, commonly sand, which adds weight and helps to keep the fibres upright; and above this is a performance infill, commonly rubber crumb particles, to provide a suitable compressible interface for player and equipment interactions.

The detailed design of $3 \mathrm{G}$ turf surfaces focuses on meeting the performance and injury risk requirements of the target sport [2]. Thus, understanding the response of the individual layers, as well as the combined surface system, to loading is key. This is recognised by the FIFA Quality Concept for football turf which uses a variety of mechanical tests to assess the performance of $3 \mathrm{G}$ turf to ensure it is safe for use [4]. Of particular importance is the vertical loading response, i.e. hardness or shock absorption, as it has been linked to both performance and injury risk $[5,6]$. From a research and development perspective, computer simulation models of the surface system, incorporating the individual layers, are used to improve understanding of the vertical loading response [7]. These models require as inputs the stress-strain properties of the individual layers of the surface system.

The most common method to characterise the stress-strain response of a material is to apply a known load and measure the resulting displacement [8]. Typically, this data is obtained using a specialist mechanical test device, such as a tensometer, due to the ease of use, controllability and repeatability they provide [9]. However, these devices are generally limited to relatively low loading rates $(\sim 3-10 \mathrm{~Hz})$ which are not representative of players interactions with sports surfaces, e.g. the heel strike of heel-toe running has a frequency of $30-50 \mathrm{~Hz}[10,11]$. This is particularly relevant for $3 \mathrm{G}$ turf where the material response is highly strain rate dependent [2]. A potential solution is to capture stress-strain data using a non-standardised test, an approach that has been used successfully to capture the high strain rate response of materials during dynamic impacts $[12,13]$.

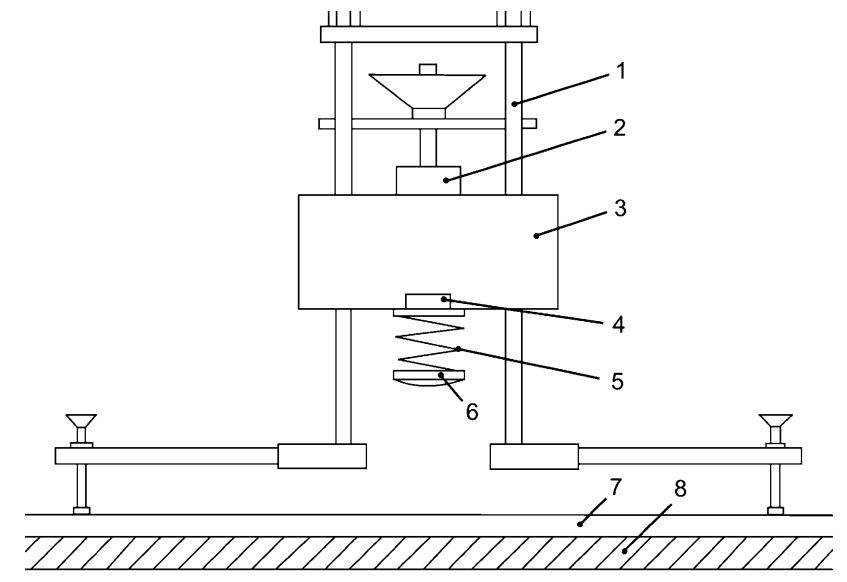

Fig. 2 The advanced artificial athlete (AAA): 1. Support frame; 2. Electric magnet; 3. Falling mass; 4. Accelerometer; 5. Spring; 6. Test foot; 7. Test surface; 8. Concrete floor [14]

The advanced artificial athlete (AAA) is a mechanical device used in the FIFA quality concept to measure and assess the stiffness response of a surface (Fig. 2) [14]. It is designed to replicate the vertical force and contact time of a heel strike during heel-toe running $[10,11,14]$. The basic principle of the device is to produce a controlled vertical impact on a surface and output the impact-rebound properties $[15,16]$. Alongside the certification outputs of force reduction, vertical deformation and energy restitution [14], further processing of the data allows the stress-strain material response throughout the contact phase to be calculated [7]. However, whilst the AAA outputs have been compared to those from its predecessor, the Artificial Athlete $[15,16]$, they have not been validated against an independent measurement system of known accuracy. For the AAA to be used to quantify the relevant strain response of sports surface systems, the accuracy of the outputs needs to be assessed.

For evaluation of multi-layered surfaces, such as $3 \mathrm{G}$ turf with a shockpad, it is important to be able to understand the deformation contributions of the individual layers to the total deformation. Hall effect sensors (HES) represent a relevant non-intrusive measurement method with the potential to assess deformation of the individual layers in a multi-layer surface system. A magnet and sensor act to indirectly measure the distance between them by producing a voltage proportional to the magnetic field at the sensor location [17]. By embedding a sensor between the carpet and shockpad layers of a $3 \mathrm{G}$ surface system (Fig. 1) and a magnet in the AAA test foot (Fig. 2), it may be possible to assess the deformation of the individual layers.

Thus, the purpose of this study was twofold: firstly, to evaluate the AAA for measurement of strain behaviour of sports surfaces; and secondly, to evaluate the potential 
use of HES to measure strain behaviour of an individual layer in multi-layer sports surfaces. To achieve the first purpose, the sample deformation output from the AAA was compared to that from the gold standard reference measurement system GOM (GOM mbH, Germany) during drop tests onto three different polymeric sports surface test samples. To achieve the second purpose, a bespoke fixture was created to allow a magnet to be embedded within an extension to the AAA test foot and a sensor grid designed to sit beneath each of the three test samples. This set-up allowed all three measurement systems to operate concurrently.

\section{Methods}

\subsection{Equipment set up and alignment}

The AAA was set up as detailed in the FIFA quality concept for football turf [14]. To replicate the human foot strike, a convex test foot is mounted to a linear spring above which a $20 \mathrm{~kg}$ mass is situated. A remote controlled electromagnet releases the mass which it is guided by a support frame throughout the drop and subsequent impact onto the surface. An accelerometer attached to the base of the falling mass records the acceleration throughout the drop (at a frequency of $9600 \mathrm{~Hz}$ ) and is used to calculate the peak force, impact and rebound velocities and vertical displacement of the $70 \mathrm{~mm}$ diameter test foot into the surface [14].

In order to apply the added instrumentation of the GOM markers and the magnets for the HES an extended test foot was attached to the existing AAA test foot (Fig. 3). The extended test foot had an identical impact face to that of the AAA but was $50 \mathrm{~mm}$ longer allowing for additional room to embed magnets and apply GOM markers. The foot was created from solid nylon such that the additional mass was negligible but also stiff enough relative to the test samples not to affect the deformation results. It was also non-conductive and, therefore, did not interfere with the HES readings. The foot was attached to the AAA through an interference press fit and adhesive tape. A solid concrete floor was used as a base for all testing and AAA calibration drops were performed directly onto this concrete floor prior to sample testing to ensure that the AAA was operating within the required limits [14]. Pilot testing on a range of test samples confirmed that the addition of the extended test foot had a negligible effect on the AAA test measurements.

The GOM setup comprised of two Photron Fastcam SA1.1 (Photron, San Diego, CA) high speed video cameras with Titanar $50 \mathrm{~mm}$ lenses used to track $5 \mathrm{~mm}$ diameter passive markers positioned on the AAA falling mass and nylon impact foot (Figs. 3a, 4). The cameras were set up according to GOM recommendations [18] and placed on a raised bar just above floor level, parallel to the plane of impact and $915 \mathrm{~mm}$ away from the measurement volume. The cameras were gen-locked and calibrated using an independently certified GOM calibration board based on a capture volume centred on the AAA falling mass and extended test foot drop zone of $300 \times 300 \times 300 \mathrm{~mm}^{3}$ (Fig. 4). This process gave a calibration deviation of less than 0.04 pixels, within the manufacturers recommendations [18]. A frame rate of $5400 \mathrm{~Hz}$ was selected to utilise the full camera resolution of $1024 \times 1024$ pixels. Shutter speed was set to $1 /$ frame rate as no motion blur was apparent at the impact speeds of approximately $1 \mathrm{~m} / \mathrm{s}$ based on
Fig. 3 a Advanced artificial athlete (AAA) with extended nylon test foot attachment. $\mathbf{b}$ Detail of extended test foot design. 1. Nylon test foot; 2. GOM markers; 3 . Socket screw; 4. Magnet; 5 . Surface sample; 6 . Hall effect sensors (HES)

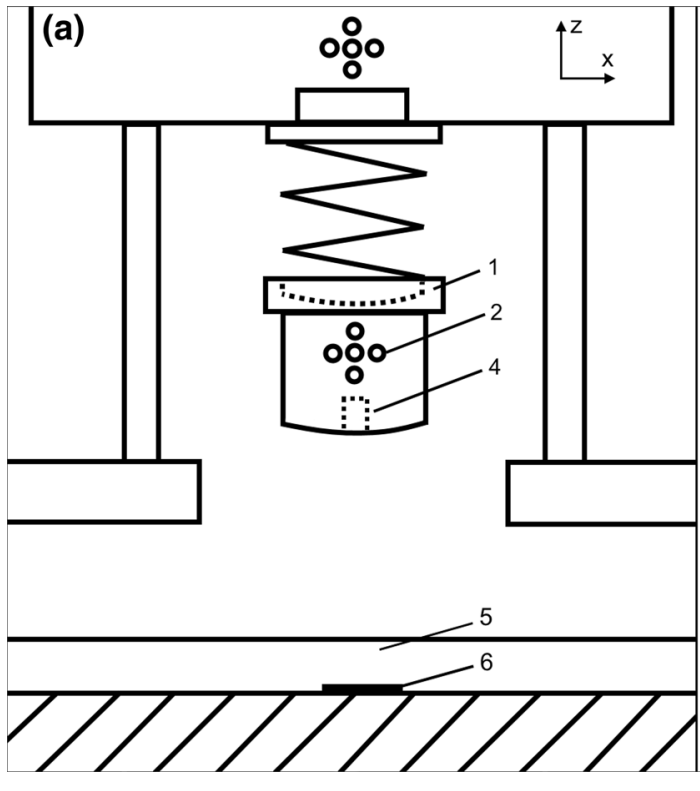

(b)

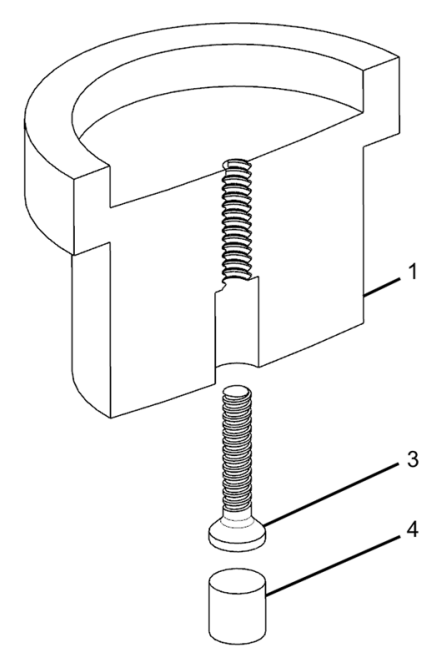

SN Applied Sciences A SPRINGer Nature journal 
Fig. 4 GOM camera set up. a Side elevation and $\mathbf{b}$ Plan view. G GOM Photron cameras; TS test sample; $L$ ARRI lighting

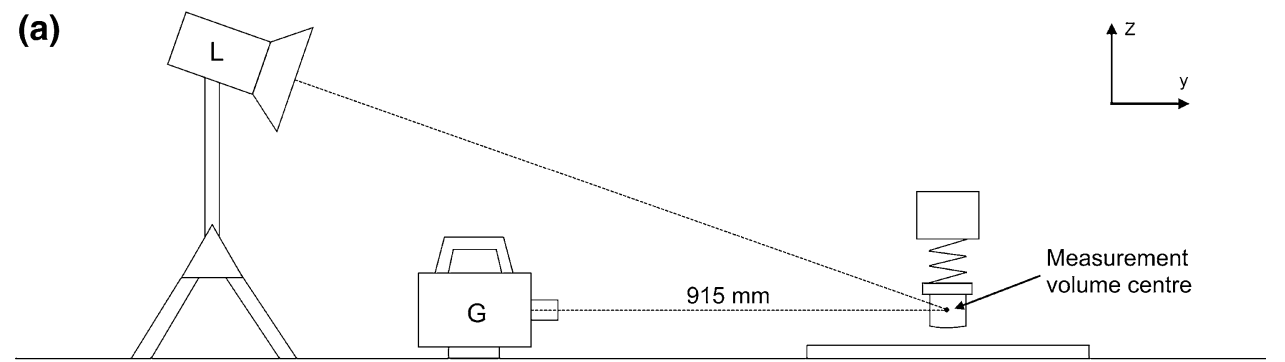

(b)

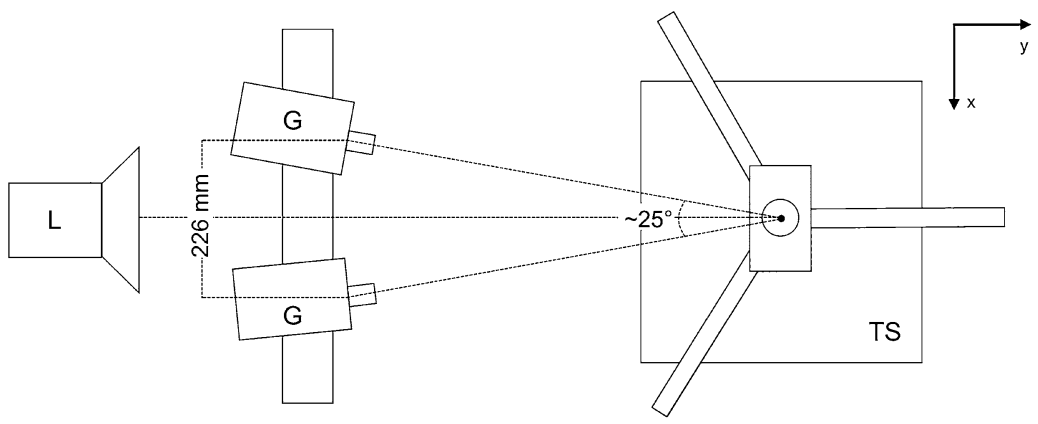

a standard AAA drop height of $55 \mathrm{~mm}$ [14]. Lens aperture was set to f/5.6 again following GOM recommendations; however, this combined with the fast shutter speed meant additional lighting was needed to ensure adequate contrast to track the markers. A single flicker-free ARRI light was directed upon the AAA extended test foot (Fig. 4). The cameras were controlled manually to record from the approximate moment of release of the AAA falling mass through to after the first rebound.

Five linear sensors (Honeywell, USA) were used to estimate the vertical position of the extended test foot. A magnet was attached to the end of a socket screw that was embedded into the extended test foot and positioned so the magnet end face sat flush with the bottom of the foot (Fig. 3b). The sensors were arranged in a quincunx configuration with $15 \mathrm{~mm}$ spacing and embedded onto a flexible printed circuit board. The board was positioned under the AAA extended test foot and securely fixed directly to the concrete floor using double-sided carpet tape (Fig. 3a). The AAA was horizontally aligned directly above the central sensor manually using the live voltage output from this sensor; alignment was taken as the horizontal position that produced maximum voltage. Alignment was assessed after every five trials and realigned if appropriate. For each trial the voltage output from all sensors was recorded via SignalExpress (National Instruments, Austin, Texas, USA) sampling at $9600 \mathrm{~Hz}$ to match that of the AAA accelerometer.

Prior to testing the central sensor was calibrated using a tensometer (Instron Electropuls E3000). The sensor board was securely fastened to a flat aluminium plate directly below the tensometer crosshead where the magnet was attached. The magnet was then lowered towards the sensor from $30 \mathrm{~mm}$ until touching in $1 \mathrm{~mm}$ increments and at each distance the corresponding voltage from the HES recorded (Fig. 5). Whilst the field sensitivity (V/T) of the HES is linear with respect to magnetic field, the sharp increase in magnetic field with respect to distance from the pole resulted in an increase of the spatial sensitivity (V/ $\mathrm{mm}$ ) as the magnet approaches the sensor. For the chosen probe current $(6 \mathrm{~mA})$ and the data acquisition system used (NI USB-6212, National Instruments, Austin, Texas) the sensor was effectively saturated at a separation of $6 \mathrm{~mm}$. This was due to the voltage generated by the HES reaching the maximum deflection of the data acquisition card. As this saturation distance was not expected to be exceeded during sample testing, the choice of probe current ensured

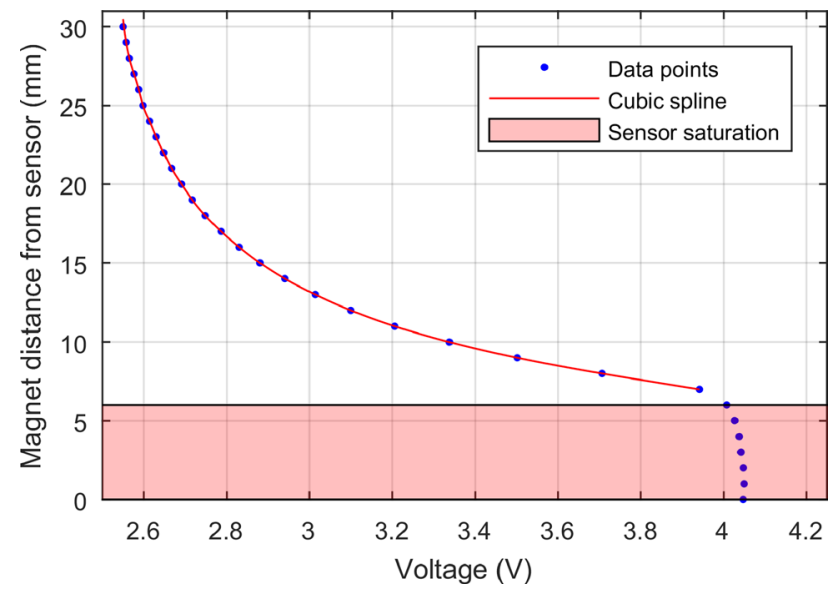

Fig. 5 Hall sensor calibration graph showing the relationship between sensor output voltage and the distance between the magnet and sensor 
maximum sensitivity over the range of the measurements presented here. Cubic spline interpolation was used to create a transfer equation to convert a recorded voltage to a magnet-sensor distance (Fig. 5).

Three test samples were chosen for testing which differed in material and thickness to provide a range of loading response conditions (Table 1). All three samples were manufactured from polymeric materials for use as or within a sports surface. The first sample was designed for racket sports with a high stiffness to allow for fast play and high ball rebound (test sample RS). The remaining two samples were FIFA approved shockpads for $3 \mathrm{G}$ turf and were, therefore, designed to absorb impact forces (test samples SP1 and SP2). All samples were at least $300 \times 300 \mathrm{~mm}$ in size. The thickness of each sample was obtained through calliper measurements taken at 10 positions on each sample while the density and material were obtained from the manufacturer datasheet.

\subsection{Protocol}

A total of 15 impacts were completed on each sample, comprising five impacts at each of three drop heights of 25,55 and $85 \mathrm{~mm}$ with the mid drop height $(55 \mathrm{~mm}$ ) representing that used in the FIFA Quality Concept for $3 \mathrm{G}$ turf [14]. A 2-min break was included between each drop to allow the sample to fully recover. The use of three drop heights resulted in a range of impact energies for evaluation of the AAA and HES.

Prior to any impacts on a sample the vertical position of the top level of the sample, i.e. the zero sample deformation position, was defined for the GOM and HES systems. For GOM this was achieved by slowly winding down the falling mass and extended test foot towards the sample until the initial point of contact between the sample and extended test foot. Visual identification of the point of contact was not possible, therefore a repeatable solution was adopted. A sheet of paper was placed between the sample and extended test foot and contact was defined at the first instance at which resistance was felt when trying to slide the paper out. Images of the position of the AAA falling mass and extended test foot were then recorded using the GOM camera set up. For the HES the sample thickness measurements were used to define the distance between the middle sensor and magnet at which initial contact with the sample occurred (Table 1).

\subsection{Data processing}

The AAA falling mass acceleration time series data for each trial was exported from the AAA software as a.csv file. The AAA software, by default, filtered the acceleration data using a 2nd order low-pass Butterworth filter at $600 \mathrm{~Hz}$ prior to export [14]. The corresponding AAA falling mass velocity and displacement data were calculated using numerical integration (trapezium rule) and force was also calculated using Newton's second law. Extended test foot displacement was calculated from the falling mass displacement and spring compression, the latter calculated using the force data and spring stiffness [14]. The extended test foot displacement was subsequently set to zero at the time of peak downward velocity, i.e. the criterion used to define the time of initial contact with the test sample [14] (Fig. 6). Sample deformation was assumed equal to extended test foot displacement during contact, with peak sample deformation corresponding to the lowest position of the extended test foot.

The GOM Correlate Professional software was used to output a.csv file containing the global positions of the markers on the falling mass and extended test foot from the video footage recorded throughout each drop and the images defining the top level of the sample. A Fourier transform analysis was performed to establish the frequency content for the marker trajectories in each trial. On this basis, a low-pass 4th order Butterworth filter was used to filter the marker trajectories before further processing, with the falling mass markers filtered at 250, 300 and $500 \mathrm{~Hz}$ and extended test foot markers at 60,50 and $50 \mathrm{~Hz}$ respectively for ascending drop heights. The sample deformation was calculated as the vertical distance of the extended test foot markers below their position defining the top level of the sample. Velocity and acceleration of the falling mass were also calculated through numerical differentiation of the displacement data (first order finite difference method) for direct comparison to the AAA data.

HES output voltages were filtered using a low-pass 4th order Butterworth filter at $50 \mathrm{~Hz}$ again determined based on the frequency content of the signals. For each trial, the

Table 1 Polymeric samples used for testing

\begin{tabular}{|c|c|c|c|c|c|c|}
\hline & Name & Manufacturer & Application & Material & Thickness (mm) & Density $\left(\mathrm{kg} / \mathrm{m}^{3}\right)$ \\
\hline RS & Regupol Kombi 1100 & BSW Berleburger & Racket sports & EPDM rubber & $11.90 \pm 0.07$ & 720 base 1100 upper \\
\hline SP1 & Regupol 6010 SP & BSW Berleburger & 3G shockpad & Polyurethane bound rubber shreds & $14.99 \pm 0.17$ & 557 \\
\hline SP2 & Re-bounce F 82.01 & Recticel & 3G shockpad & Bound polyurethane foam & $24.07 \pm 0.14^{*}$ & 310 \\
\hline
\end{tabular}

*2 layers of shockpad used 

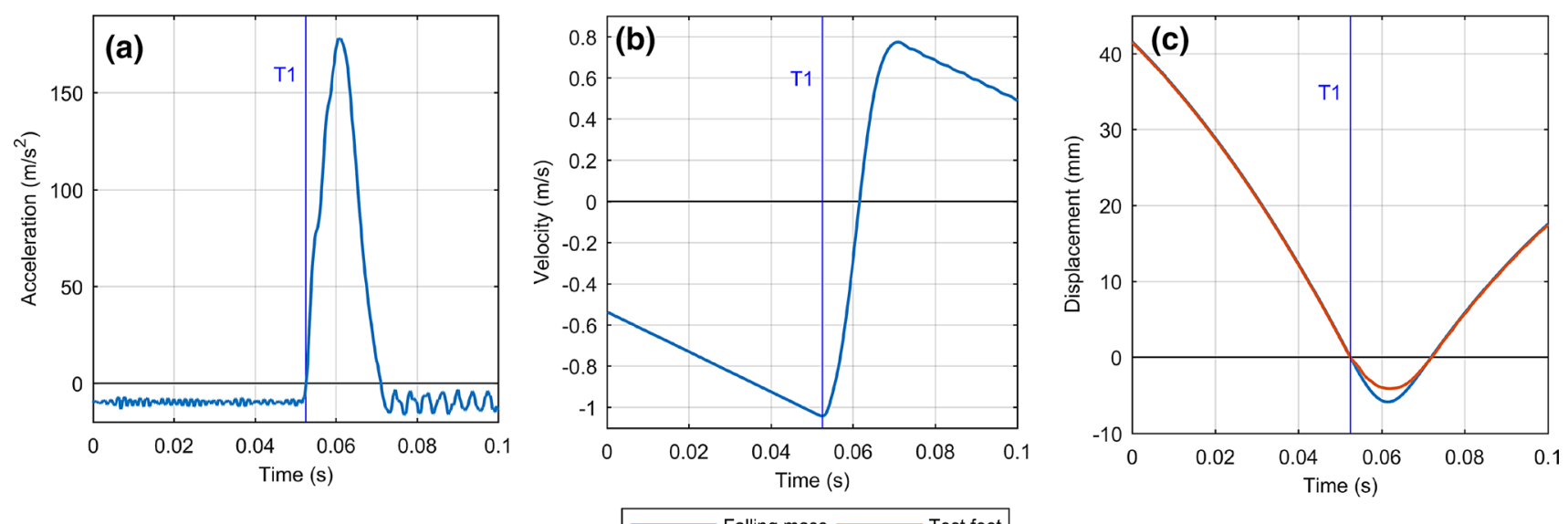

Fig. 6 Typical a acceleration b velocity and $\mathbf{c}$ displacement data from the advanced artificial athlete (AAA) during an impact onto the SP1 shockpad. T1 represents the time of peak downward velocity and the time at which the extended test foot displacement was set to zero (assumed initial contact with the sample). Extended test foot displacement was calculated by subtracting the spring compression from the falling mass displacement

relevant, regression analysis was conducted to explore the scope of correcting the AAA and/or HES measurements to remove the systematic bias and give improved absolute agreement with the GOM measurements. To compare the full sample time series data throughout the contact phase root mean square differences (RMSD) were calculated between AAA-GOM and HES-GOM in falling mass acceleration and velocity as well as sample strain. Absolute values of RMSD were reported for each variable, however, to allow for comparison across multiple variables, these values were also normalised by dividing by the peak values of each variable across the time series and reporting a normalised RMSD (expressed as a percentage of the peak value of the variable). All statistical analysis was conducted in Matlab with significance set at $p<0.05$.

To assess whether the accuracy of the AAA and HES strain measures were sufficient for material characterisation, it was recognised that when recording material load-deformation data under high loading rates, oscillations (noise) are typically observed in the resulting stress-strain plots. However, provided these oscillations are relatively small, the (real) material stress-strain response can be discerned and used for material characterisation [20]. On this basis, a threshold root mean square deviation (RMSD) of $10 \%$ of the peak strain was selected as an appropriate criterion indicating that the real strain response had been captured with sufficient accuracy. (closer to zero indicates lower systematic bias) whilst the $95 \%$ confidence intervals ( \pm 1.96 standard deviations) gives an indication of the random differences between the two measurement systems (larger confidence intervals indicates larger random differences). To further assess the systematic bias between systems, repeated measures t-tests were also conducted on the peak sample deformations. Where

\section{SN Applied Sciences}




\section{Results}

All three measurement systems were successful in capturing data for all trials with the exception of the $85 \mathrm{~mm}$ drops onto the RS sample where only one successful capture was obtained for the HES due to the sensors breaking. Consequently, this trial was disregarded in the data analysis and the results presented below for the HES are based on the remaining 40 trials.

\subsection{AAA evaluation}

Outputs from AAA and GOM showed good qualitative agreement in the falling mass acceleration and velocity profiles (Fig. 7). Peak acceleration and downward velocity were within $5 \%$ and $1 \%$ respectively and the RMSD between acceleration curves was $4.0 \pm 0.6 \mathrm{~m} / \mathrm{s}^{2}$ and between velocity curves was $0.014 \pm 0.003 \mathrm{~m} / \mathrm{s}$ (Table 2). Key metrics from the contact phase showed poor agreement in falling mass displacement (AAA 2-3 mm lower), peak sample deformation (AAA 1-3 mm lower) and time to peak deformation (AAA 1.0-4.5 ms lower) but better agreement in spring compression ( $\leq 0.4 \mathrm{~mm}$ difference) (Table 3).

The Bland-Altman analysis confirmed that the AAA under-estimated peak sample deformation for all conditions (Fig. 8a). The systematic bias increased as peak sample deformation increased; from approximately $1 \mathrm{~mm}$ for the lowest peak deformations to $3 \mathrm{~mm}$ at the highest peak deformations (giving a mean difference of $2.4 \mathrm{~mm}$ ), with the limits of agreement $( \pm 1.3 \mathrm{~mm}$ ) largely defined by this trend rather than by random differences. Given the systematic nature of these errors in AAA sample deformation and good overall agreement between the fundamental measurements of acceleration and velocity, the main source of error can be identified as the definition of initial contact with the sample. For the AAA this was the time of peak downward velocity and for GOM it was based on the independently measured position of the top level of the sample. These times systematically differed (Fig. 7; Table 3) leading to systematic differences in the absolute vertical position of zero sample deformation and, therefore, in peak sample deformation (Fig. 7c). Peak downward velocity occurred after contact had been made with the sample (Fig. 7b) (where the acceleration crossed $0 \mathrm{~m} / \mathrm{s}^{2}$ rather than first deviating from $-9.81 \mathrm{~m} / \mathrm{s}^{2}$ ) leading to the AAA systematically under-predicting sample deformation (Fig. 7c).

Given that the differences between the AAA and GOM sample deformation values were predominantly systematic, a regression analysis between the two was conducted to explore the feasibility of correcting the AAA data to give closer agreement with the gold standard. As highlighted above, the main source of error came from the incorrect identification of the time of initial contact with the sample (Fig. 7) and the magnitude of error was a function of the peak sample deformation (Fig. 8). Thus, the correction was based on altering the AAA time of initial contact to better match that defined by GOM as a function of peak acceleration. A plot of peak acceleration against time difference for all 45 trials supported an exponential regression equation to be fitted with a RMSD of only 0.27 ms (Fig. 9):

$(T 1-T 2)=6.438 \times e^{-0.0058 \times a_{p k}}$

where $T 1$ is the time of peak downward velocity (in ms), $T 2$ is the time of initial contact with the sample (in ms) and $a_{P K}$ is the peak acceleration (in $\mathrm{m} / \mathrm{s}^{2}$ ). Thus, from the AAA
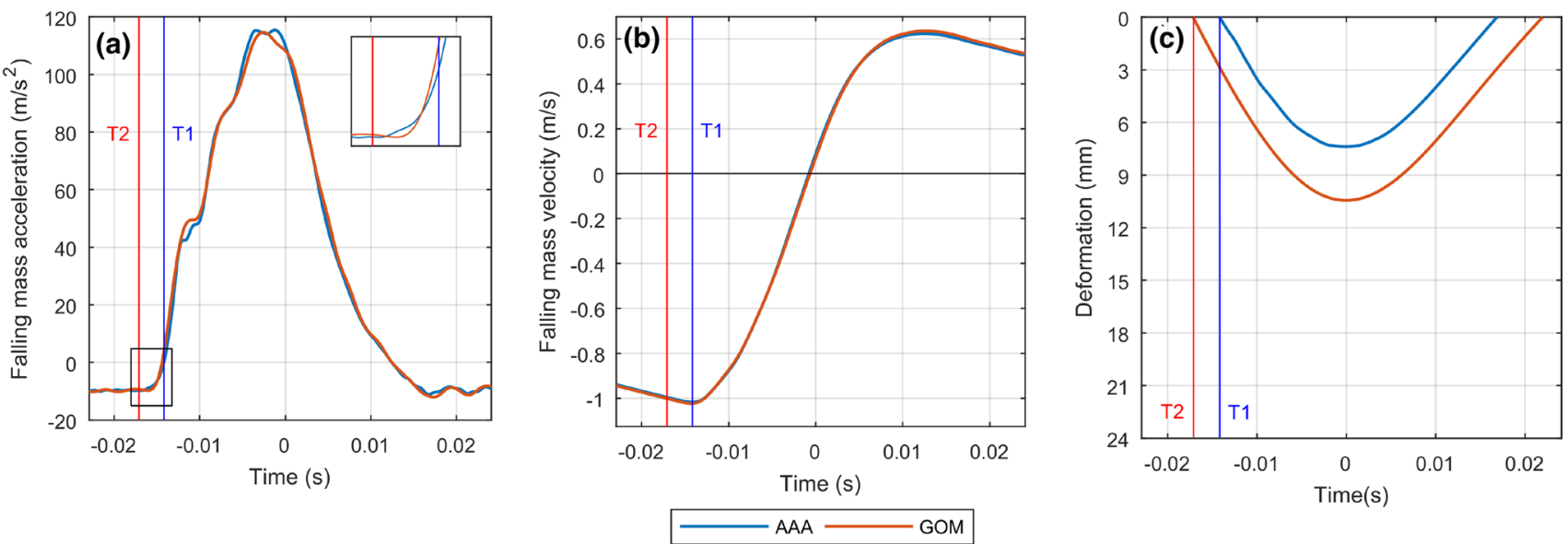

Fig. 7 Typical a falling mass acceleration $\mathbf{b}$ falling mass velocity and c sample deformation from advanced artificial athlete (AAA) and GOM for the SP2 shockpad. The blue vertical line represents the time of peak downward velocity (T1) whilst the red vertical line represents the time of initial contact with the sample based on the GOM marker positional data (T2) 
Table 2 Root mean square difference (RMSD) results

\begin{tabular}{|c|c|c|c|c|c|c|}
\hline \multirow[t]{2}{*}{ Sample } & \multirow[t]{2}{*}{ Drop height (mm) } & \multirow{2}{*}{$\begin{array}{l}\text { Acceleration }\left(\mathrm{m} / \mathrm{s}^{2}\right) \\
\text { AAA-GOM }\end{array}$} & \multirow{2}{*}{$\begin{array}{l}\text { Velocity }(\mathrm{m} / \mathrm{s}) \\
\text { AAA-GOM }\end{array}$} & \multicolumn{3}{|c|}{ Sample strain (-) } \\
\hline & & & & AAA-GOM & AAAnew-GOM & HES-GOM \\
\hline \multirow[t]{5}{*}{ RS } & 25 & $6.0 \pm 0.7$ & $0.012 \pm 0.002$ & $0.085 \pm 0.003$ & $0.050 \pm 0.003$ & $0.057 \pm 0.005$ \\
\hline & 55 & $6.2 \pm 1.2$ & $0.016 \pm 0.003$ & $0.101 \pm 0.005$ & $0.033 \pm 0.001$ & $0.059 \pm 0.002$ \\
\hline & 85 & $9.7 \pm 1.2$ & $0.031 \pm 0.003$ & $0.099 \pm 0.013$ & $0.038 \pm 0.005$ & - \\
\hline & All & $7.3 \pm 2.0$ & $0.020 \pm 0.009$ & $0.095 \pm 0.011$ & $0.040 \pm 0.008$ & $0.058 \pm 0.004$ \\
\hline & $\%$ of peak & $3.0 \pm 0.7 \%$ & $2.2 \pm 0.5 \%$ & $34.9 \pm 2.0 \%$ & $15.1 \pm 4.5 \%$ & $16.2 \pm 10.4 \%$ \\
\hline \multirow[t]{5}{*}{ SP1 } & 25 & $1.5 \pm 0.3$ & $0.007 \pm 0.001$ & $0.130 \pm 0.001$ & $0.016 \pm 0.004$ & $0.051 \pm 0.004$ \\
\hline & 55 & $2.3 \pm 0.4$ & $0.011 \pm 0.002$ & $0.143 \pm 0.007$ & $0.020 \pm 0.005$ & $0.065 \pm 0.004$ \\
\hline & 85 & $2.8 \pm 0.4$ & $0.017 \pm 0.002$ & $0.143 \pm 0.005$ & $0.037 \pm 0.006$ & $0.055 \pm 0.001$ \\
\hline & All & $2.3 \pm 0.6$ & $0.012 \pm 0.005$ & $0.139 \pm 0.008$ & $0.024 \pm 0.011$ & $0.058 \pm 0.007$ \\
\hline & $\%$ of peak & $1.3 \pm 0.3 \%$ & $1.5 \pm 0.3 \%$ & $29.4 \pm 2.7 \%$ & $5.0 \pm 1.8 \%$ & $12.0 \pm 1.5 \%$ \\
\hline \multirow[t]{5}{*}{ SP2 } & 25 & $1.8 \pm 0.3$ & $0.006 \pm 0.001$ & $0.089 \pm 0.003$ & $0.010 \pm 0.001$ & $0.015 \pm 0.004$ \\
\hline & 55 & $2.2 \pm 0.1$ & $0.009 \pm 0.001$ & $0.095 \pm 0.003$ & $0.020 \pm 0.003$ & $0.019 \pm 0.003$ \\
\hline & 85 & $3.2 \pm 0.2$ & $0.012 \pm 0.001$ & $0.094 \pm 0.003$ & $0.020 \pm 0.003$ & $0.016 \pm 0.002$ \\
\hline & All & $2.1 \pm 1.7$ & $0.006 \pm 0.007$ & $0.090 \pm 0.086$ & $0.009 \pm 0.011$ & $0.013 \pm 0.012$ \\
\hline & $\%$ of peak & $2.2 \pm 0.5 \%$ & $1.4 \pm 0.2 \%$ & $22.4 \pm 3.4 \%$ & $3.9 \pm 0.8 \%$ & $4.0 \pm 0.9 \%$ \\
\hline Overall & & $4.0 \pm 0.6$ & $0.014 \pm 0.003$ & $0.109 \pm 0.004$ & $0.027 \pm 0.005$ & $0.043 \pm 0.003$ \\
\hline Overall \% of peak & & $2.2 \pm 0.9 \%$ & $1.7 \pm 0.5 \%$ & $28.9 \pm 5.8 \%$ & $8.0 \pm 5.8 \%$ & $10.7 \pm 7.9 \%$ \\
\hline
\end{tabular}

RMSD in falling mass acceleration and velocity between advanced artificial athlete (AAA) and GOM and in sample strain between AAA and GOM and HES and GOM. All calculated over the contact phase as defined by the GOM

accelerometer values for $T 1$ and $a_{P K}$, the corrected time of initial contact with the sample (T2) can be calculated.

To further assess the applicability of this correction, the AAA outputs were re-calculated including this correction to the time of initial contact (AAAnew in Tables 2, 3; Figs. 8b, 10). In the Bland-Altman analysis this reduced the systematic bias (mean difference) from $2.4 \mathrm{~mm}$ down to $0.1 \mathrm{~mm}$ and the random differences (limits of agreement) from $\pm 1.3 \mathrm{~mm}$ down to $\pm 0.7 \mathrm{~mm}$ (Fig. 8). The improved agreement was also reflected in the strain data where the strain RMSD compared to GOM decreased from $0.11 \pm 0.02$ to $0.03 \pm 0.01$ (Table 2; Fig. 10). Upon breakdown, both shockpad samples met the criterion for material characterisation as their respective RMSDs were within $10 \%$ of the peak GOM strain (SP1 $5 \pm 2 \%$ and SP2 $4 \pm 1 \%$ ). Despite an improvement in RMSD, the RS sample did not fall within the required criterion for material characterisation (15 $\pm 5 \%$; Table 2$)$.

\subsection{HES evaluation}

Outputs from HES and GOM showed good overall agreement in peak sample deformation (Table 3). The Bland-Altman analysis revealed that the peak sample deformation from the HES had no significant systematic bias (mean difference $0.2 \mathrm{~mm}$ ) and small random differences of $\pm 0.3 \mathrm{~mm}$ (Fig. 11). There was a trend for the HES to over-predict peak sample deformation for the stiffer response conditions (smallest peak deformations) but under-predict peak sample deformation for the least stiff response conditions (largest peak deformations). Similarly, time to peak sample deformation was over-estimated for the RS and SP1 samples (by $1.5-2 \mathrm{~ms})$ but was well matched by the SP2 sample $(<0.5 \mathrm{~ms}$ difference; Table 3; Fig. 12). This was also reflected in the sample strain data where the SP2 sample produced a better fit $(0.017 \pm 0.003)$ compared to the SP1 $(0.058 \pm 0.007)$ and RS $(0.058 \pm 0.004)$ samples (Table 2; Fig. 12). Only SP2 met the criterion for material characterisation with a strain RMSD of $4 \pm 1 \%$ of peak strain with the remaining samples $\geq 12 \%$. Linked to this, despite the good agreement in peak sample deformation, at initial contact (based on GOM) the differences were much bigger with sample deformation overpredicted by at least $1 \mathrm{~mm}$ for both the RS and SP1 samples, while agreement was much better for the SP2 sample. RS and SP1 samples both deformed in the range $7-15 \mathrm{~mm}$ from the sensor. In contrast the SP2 sample deformed 11-24 mm from the sensors and, therefore, operated in a lower sensitivity region $(\sim 0.16 \mathrm{~V} / \mathrm{mm})$ compared to the other two samples $(\sim 0.04 \mathrm{~V} / \mathrm{mm})$ (Fig. 5).

\section{Discussion}

Computer simulation models are a useful tool to improve understanding related to the vertical loading response, and hence cushioning effects, of sports surface systems 


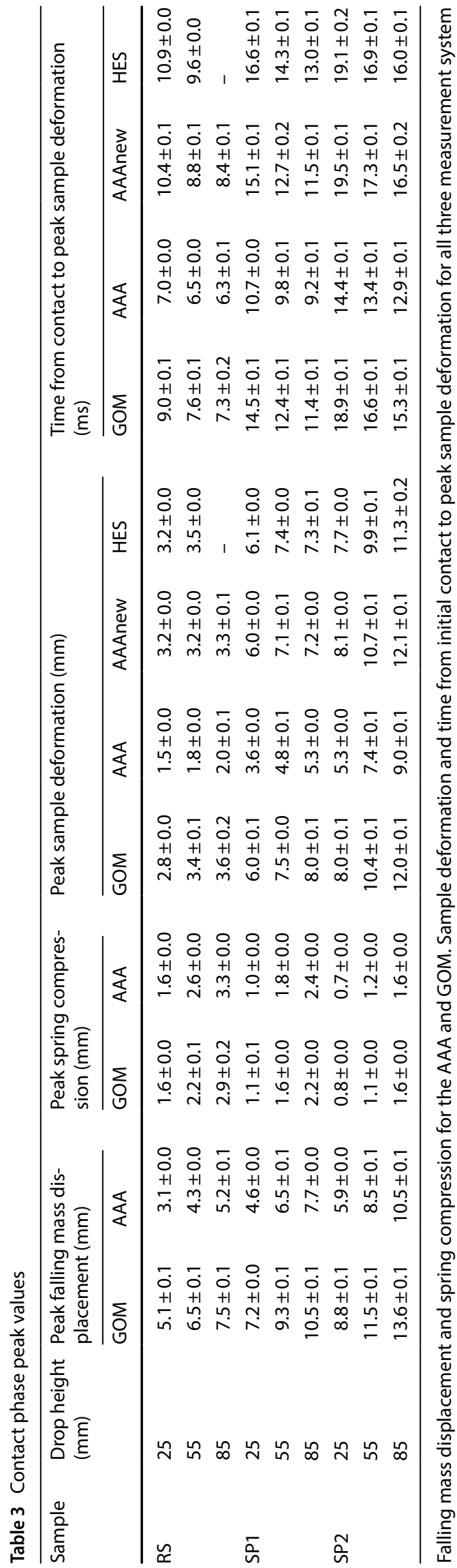

[7]. However, characterising the component material stress-strain properties under the high loading rates typical of player and equipment interactions with sports surfaces using traditional test methods is challenging. The AAA is a mechanical drop test device specifically designed to replicate certain features of player-surface interactions, including the high loading rates. However, its ability to provide accurate stress-strain characteristics throughout surface contact has yet to be investigated. Hence, the first objective was to evaluate the use of the AAA to measure the strain behaviour of sport surfaces. Whilst the AAA provides data on the overall deformation of a sport surface system, it cannot provide information on the contribution of the individual layers to this overall deformation in a multi-layer system (as often used in sport). Measurement of the individual layer deformations is relevant to surface system research and development through either physical testing or virtual modelling. Hall effect sensors provide a non-intrusive distance measurement method with potential to be applied to intra-layer deformation measurement. Thus, the second objective was to evaluate the potential of HES to support measurement of the strain behaviour of individual layers in multi-layer surface systems. Three test samples and three AAA drop heights were used to provide a wide range of sample responses to dynamic loading (confirmed by the wide range in peak falling mass decelerations from 60 to $330 \mathrm{~m} / \mathrm{s}^{2}$ and in peak sample deformations from 2.8 to $12.0 \mathrm{~mm}$ ) while simultaneously providing good control over the test conditions to support the evaluation of the AAA and HES measurement systems.

The optical measurement system GOM was used as the gold standard reference for surface sample deformation and strain. It has a manufacturer reported accuracy of $25 \mu \mathrm{m} / \mathrm{m}$ field of view which was also demonstrated in a recent independent study [21]. Measurement volume, camera set-up and calibration procedures similar to Leach [21] were applied in this study to support its use for the evaluation of the deformation and strain outputs from the AAA and HES measurement systems. It should be noted that while there was high confidence in the accuracy of the GOM positional data as the gold standard, numerical differentiation was required to obtain GOM velocity and acceleration. Consequently, confidence in these values was lower and, on this basis, GOM velocity and acceleration were considered for comparative purposes only.

Strain, calculated from the AAA/HES sample deformation data and sample thickness, was deemed suitable for material characterisation if the RMSD (compared to the GOM strain data) was less than $10 \%$ of the peak strain. Previous studies investigating impacts under high loading rate conditions found that material characterisation could successfully be achieved if the main features of the material response could be discerned from the stress-strain 


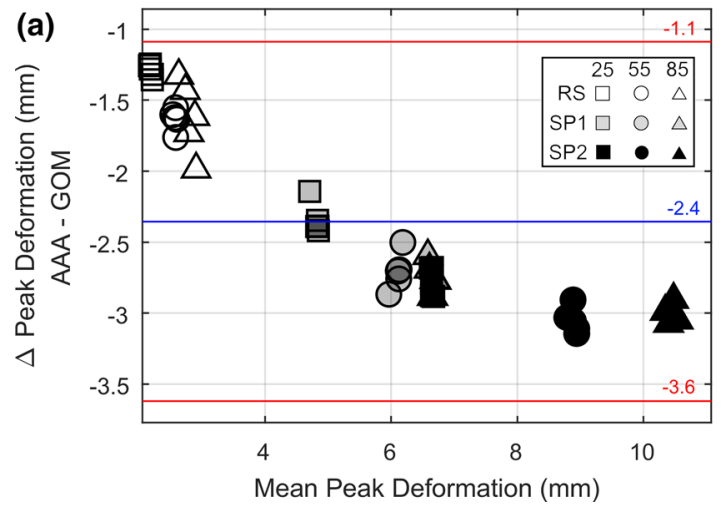

Fig. 8 Bland-Altman plot comparing the GOM gold standard to a the advanced artificial athlete (AAA) values of peak sample deformation and $\mathbf{b}$ the new AAA values of peak sample deformation cal-

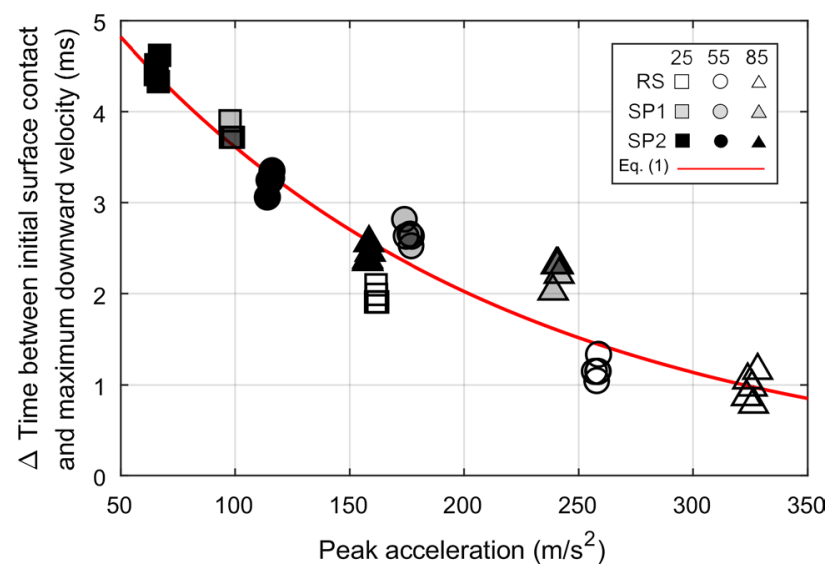

Fig. 9 Relationship between peak acceleration and time difference between initial contact with the sample and peak downward velocity (T2-T1). The red line displays the exponential line of best fit [Eq. (1)]

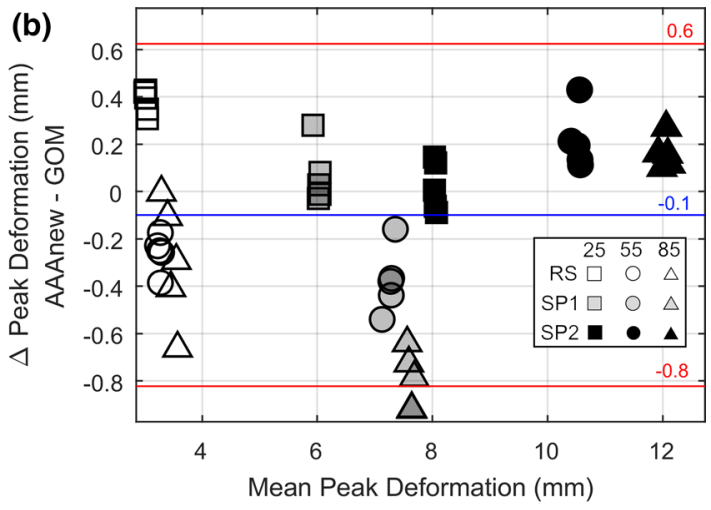

culated using regression Eq. (1). The horizontal blue line represents the mean difference and the red lines the lower and upper quartiles (95\% confidence intervals)

data [20]. However, this assessment was not quantified and, therefore, an estimated criterion of strain RMSD less than $10 \%$ of the peak strain was proposed here, which visually appeared to meet the qualitative criterion detailed above. Expressing the RMSD relative to the peak strain provided a relevant means of accounting for the differences in sample thickness. Calculating the strain RMSD over the whole stress-strain curve was considered to be important since the samples used in this study, along with many of the materials used in $3 \mathrm{G}$ surfaces, are hyperelastic suggesting that the rate of change in strain as well as energy loss in recovery are important features to capture.

Poor agreement was obtained between AAA and GOM for peak sample deformation and strain. The AAA underpredicted peak deformation by between 25 and $47 \%$ and the overall average strain RMSD was $29 \%$ of peak strain (Tables 2, 3; Figs. 8a, 10). With much better agreement in
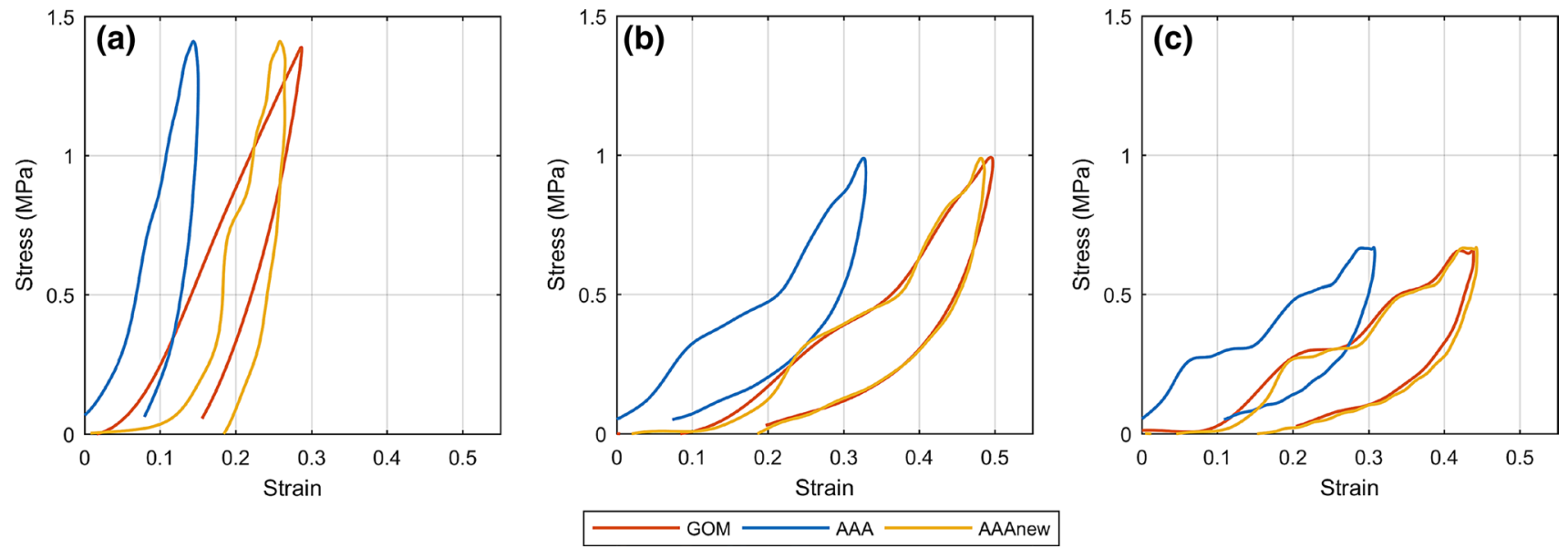

Fig. 10 Typical sample stress-strain plots derived from the advanced artificial athlete (AAA), the corrected AAA and the GOM data for the $55 \mathrm{~mm}$ drop height onto a RS, b SP1 and c SP2 samples 


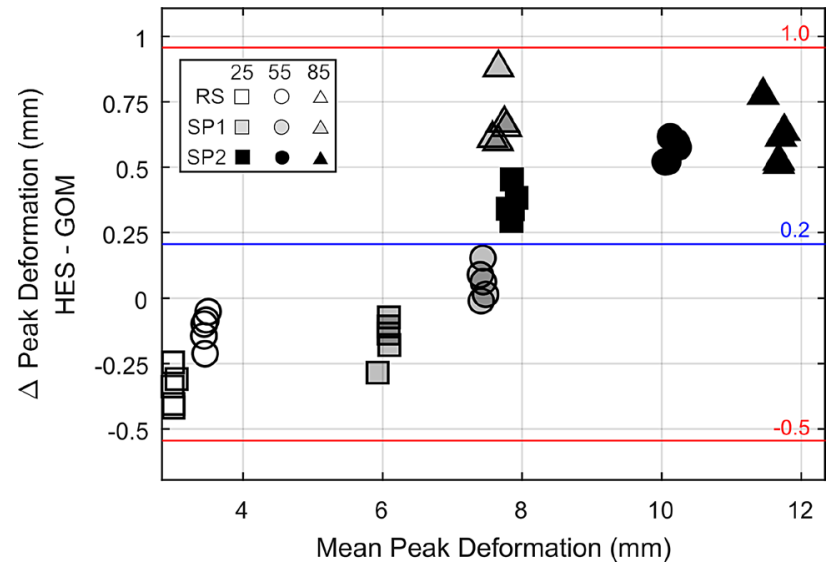

Fig. 11 Bland-Altman plot comparing the Hall effect sensor (HES) values of peak sample deformation to the GOM gold standard. The horizontal blue line represents the mean difference and the ref lines the lower and upper quartiles (95\% confidence intervals)

acceleration and velocity data, the poor agreement was identified as resulting from the method used by the AAA to determine the time of initial contact of the extended test foot with the sample, i.e. the zero sample deformation conditions. The AAA used the time of peak downward velocity of the falling mass as initial contact corresponding to the acceleration crossing $0 \mathrm{~m} / \mathrm{s}^{2}$; however, initial sample contact occurs at an earlier time point where the acceleration first deviates from gravitational acceleration with the GOM time of initial contact providing a much better estimate of this instant (Fig. 7). As expected based on the foregoing, the error in AAA time of initial contact increased for the more compliant test sample, characterised by lower peak impact decelerations (Fig. 9); however, given its systematic nature correction methods were explored.
A correction method for the AAA time of initial contact was developed based on fitting an exponential regression equation relating the AAA falling mass peak deceleration to the time difference between GOM initial contact and the original AAA initial contact based on peak downward velocity (Fig. 9). The applicability of this regression equation was explored by re-calculating the AAA sample deformation data (AAAnew) and repeating the comparison to the GOM sample deformation and strain. The correction resulted in much better agreement; for peak sample deformation there was no significant systematic bias (mean difference of $<2 \%$ or $<0.1 \mathrm{~mm}$ ) and random differences of $\pm 0.7 \mathrm{~mm}$ ( $95 \%$ confidence intervals). The strain RMSD was reduced to $8 \%$ of peak strain (Tables 2, 3; Figs. $8 \mathrm{~b}$, $10)$, thus meeting the criterion for material characterisation. The correction appeared to work equally across all test samples and drop heights for peak sample deformation; however, for strain RMSD, tests onto sample RS gave higher RMSDs ( $15 \pm 5 \%$ of peak strain) than those onto the remaining two samples $(\leq 5 \%)$, largely due to the smaller (unloaded) thickness of this sample and smaller peak deformations. Given the empirical nature of the correction method, the recommendation is that it should only be applied to impacts with peak falling mass decelerations in the range over which it was developed $\left(60-330 \mathrm{~m} / \mathrm{s}^{2}\right)$. With this caveat in place, the results support the use of the AAA drop test, with the inclusion of the correction to the time of initial contact, as a suitable method for measuring the deformation of sports surface systems under high loading rate conditions, giving sub $1 \mathrm{~mm}$ accuracy. In terms of strain measurement, the errors naturally scale with the surface or layer thickness indicating that it should be used with caution for thin, stiff surfaces experiencing peak deformations of $<4-5 \mathrm{~mm}$.
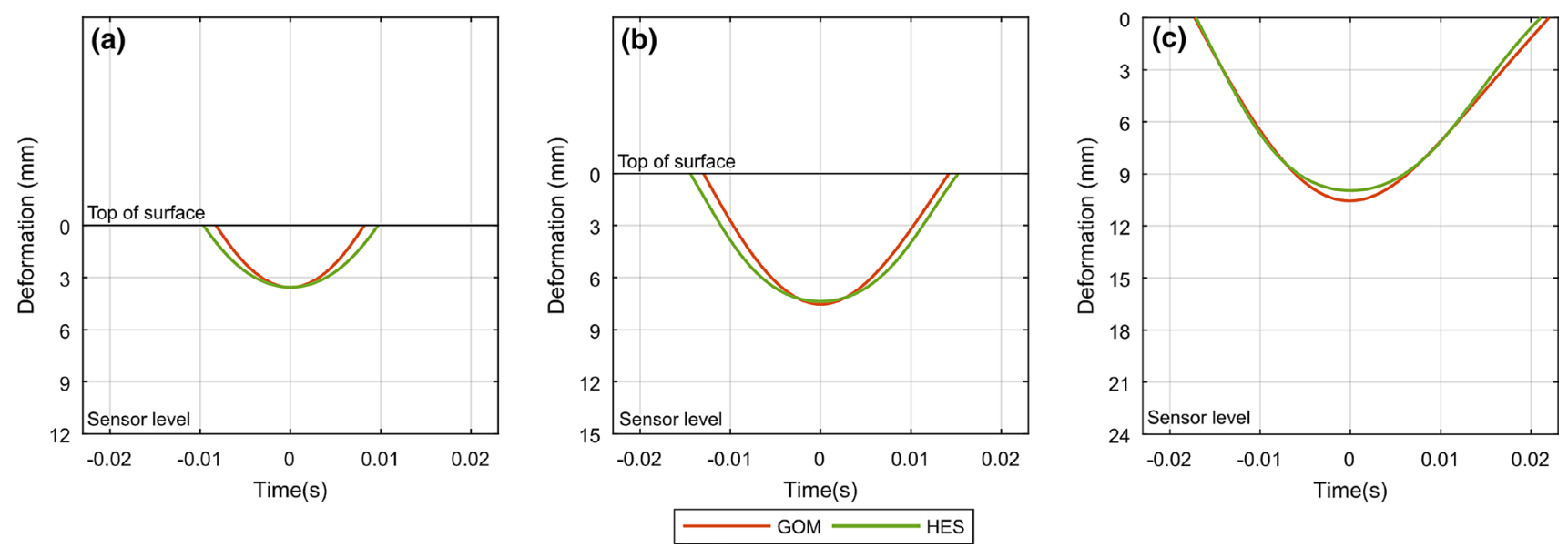

Fig. 12 Typical sample deformation profiles for the Hall effect sensors (HES) and GOM systems for a RS, b SP1 and c SP2 samples. The y-axis range spans from the top of the sample $(0 \mathrm{~mm})$ to the vertical position of the sensor 
Very good agreement was obtained between HES and GOM for peak sample deformation but poorer agreement for strain RMSD (Tables 2, 3; Figs. 11, 12). Peak sample deformation systematic bias and random differences were $0.2 \mathrm{~mm}$ and $\pm 0.3 \mathrm{~mm}$ ( $95 \%$ confidence intervals) respectively, while the overall average strain RMSD was $11 \%$ of peak strain, just outside the criterion for material characterisation. There were, however, clear inter-sample differences in the level of agreement; notably, while SP2 sample had the lowest strain RMSD (4\%, i.e. within the criterion for material characterisation), it had the highest mean difference in peak sample deformation (underestimated by $0.5 \mathrm{~mm}$ ).

To explore the reasoning behind these observations, initially the HES calibration curve was considered with respect to measurement sensitivity (Fig. 5). Differences in the three sample thicknesses and peak deformations resulted in each sample deformation range falling within a different range on the HES calibration curve and, therefore, within a different sensitivity range. SP1 and RS samples were thin and stiff, relative to SP2, thus the magnet was closer to the sensor board during deformation, resulting in a higher average sensitivity $(0.15$ and $0.17 \mathrm{~V} / \mathrm{mm}$ respectively). In contrast, SP2 was double the thickness and had a sample deformation range that sat on the steeper part of the calibration curve corresponding to a much lower average sensitivity $(0.04 \mathrm{~V} / \mathrm{mm})$. On this basis, it might be expected that the HES errors would increase with increasing magnet-sensor distance; however, this wasn't observed in the test results indicating that sensor sensitivity wasn't the main factor limiting the performance of the HES measurement system. Given the relatively limited range of magnet to sensor distance over which a HES operates (particularly with high sensitivity), these results reinforce the importance of careful selection of magnet properties for the specific application.

To further explore the errors in the HES measurements, horizontal movement of the AAA device during testing leading to poor vertical alignment between the magnet and sensor was also considered. The HES system relied on an accurate manual vertical alignment between the sensor and magnet to precisely maintain them on the same vertical axis. Whilst the alignment protocol was completed at the start of every set of five trials (sample and drop height), vibration of the AAA device during each drop may have caused some horizontal movement of the device relative to the sensor which was hard to perceive visually. Analysis of the GOM markers on the extended test foot in the horizontal plane across each set of five trials revealed a small permanent horizontal displacement of the extended test foot of up to $0.4 \mathrm{~mm}$. Although the magnitude of this horizontal displacement is too small to substantially explain the HES test sample results, it reinforces the importance of careful vertical alignment of the magnet over the sensor to maximise the accuracy of the HES deformation and strain measurements.

The AAA is used globally for certification of a sport surface system in comfort and regard safety, for example in the FIFA Quality Concept for football turf [14]. The three variables derived from the AAA test used to define surface performance are; force reduction, vertical deformation and energy restitution. It is relevant to consider the implications of the results of this study to these outputs. The calculation of force reduction relies on the peak falling mass deceleration and, therefore, is unaffected by this study findings. Vertical deformation is the equivalent of the peak sample deformation reported in this study and; the AAA has been demonstrated to systematically underpredict this variable due to a systematic error in the time of initial contact with the surface/sample. Given that the acceptable limits of force reduction in the FIFA standard correspond to peak falling mass decelerations in the range $90-145 \mathrm{~m} / \mathrm{s}^{2}$, the corresponding error in time of initial surface contact can then be estimated as 3-4 ms which may result in an under-estimation of vertical deformation by as much as $2-3 \mathrm{~mm}$. It should be noted the FIFA limits of acceptability for vertical deformation of a football turf surface is $4-11 \mathrm{~mm}$. Although the potential error of 2-3 mm may appear large, the football turf standards are based on benchmarking to good quality natural grass such that the current limits are empirical rather than based on any fundamental absolute values. Hence systematic bias may not be an issue in regard to surface safety implications. Notably, the Quality Concept requires vertical deformation to be reported to the nearest $0.5 \mathrm{~mm}$ which corresponds to the standard deviation of the random errors observed in this study. Energy restitution is the ratio of the falling mass energy immediately post-impact to pre-impact. Its calculation is based on the peak upward velocity (postimpact) and peak downward velocity (pre-impact) of the falling mass. This study has demonstrated that the peak downward velocity occurs after impact (by 3-4 ms for surfaces tested within the Quality Concept) and by a similar reasoning, the peak upward velocity will occur before the extended test foot loses contact with the surface. For the three surface samples and three drop heights used in this study, applying the erroneous peak velocity criterion to estimate pre- and post-impact velocities resulted in energy restitution values $4.0 \pm 1.8 \%, 2.0 \pm 3.0 \%$ and $8.4 \pm 4.5 \%$ smaller (for RS, SP1 and SP2 respectively) than using the correct pre- and post-impact velocity method. At present, however, the FIFA standard requires energy restitution to be reported, but does not stipulate limits of acceptability. In contrast the World Rugby standard [22], based on the same AAA test method and empirical basis, stipulates an acceptable range of energy restitution as $20-50 \%$. 
Although the AAA has replaced its predecessor, the Artificial Athlete (AA), in the certification testing of sports surfaces, the latter is still widely used in the hardness testing of sports surfaces. Previous comparisons of the force reduction and vertical deformation values from the two devices have indicated no significant differences [15]. However, there are fundamental differences in the design and measurement systems employed by the two devices; notably, for the AA the falling mass is dropped onto the spring and test foot which start stationary on top of the surface, with the load cell and movement sensors embedded in the test foot rather than the falling mass (Fig. 2). These differences indicate that the current results for the AAA cannot be extrapolated to the AA and a separate evaluation of the latter is needed to assess its suitability for measuring strain behaviour of polymeric sports surfaces.

Some limitations in this study should be recognised. Firstly, only deformation and strain measurements from the AAA have been evaluated and not the stress data. This would require a further study with the selection of an appropriate gold standard measurement system for stress. Secondly, the HES was not tested within a multilayer surface system. The sensor was attached to a rigid concrete floor, whereas in a multi-layer system it would be positioned at a more deformable interface between layers, e.g. between the shockpad and carpet backing for a $3 G$ turf surface system. The performance of the HES under these conditions needs to be investigated to fully evaluate its potential for reliable intra-layer deformation measurements in multi-layer sports surfaces. However, the accuracy results for HES deformation and strain in this study support pursuing this next stage of evaluation and validation.

\section{Conclusions}

This study has demonstrated the applicability of two measurement systems for utilisation in future numerical modelling investigations into the vertical loading response of sports surface systems under dynamic impact loading. While direct application of the AAA to measure deformation and strain behaviour of sports surface systems is not recommended with large (predominantly systematic) errors in peak deformation and strain, the corrected AAA method, based on Eq. (1), can be considered as an acceptable means to measure peak deformation and strain behaviour of sports surface systems under high loading rates. Peak deformation errors remained relatively consistent $(\sim 0.7 \mathrm{~mm})$ across test conditions, while strain RMSD increased with decreasing sample thickness and peak deformation; suggesting that even this corrected AAA method should be used with caution for samples undergoing small peak deformations ( $<4-5 \mathrm{~mm})$. HES should be used with caution to explore intra-layer deformations in multi-layer sport surface systems. This system gave small errors in peak deformation $(0.5 \mathrm{~mm})$, while strain RMSD again increased with decreasing sample thickness and peak deformation. However, the strain errors were slightly larger than for the corrected AAA such that only the thickest and most compliant surface sample gave strain RMSD which satisfied the material characterisation criterion. Further sources of error, such as the effect of sample permeability on the magnetic field and the accuracy of the voltage to distance transfer equation, should be explored before further testing.

Authors' contributions DC, PF and SF conceived the presented idea. All authors were involved in the experimental design. DC conducted the experiments and processed the data. DC, PF and SF wrote the manuscript. All authors reviewed the manuscript and approved the final version.

Availability of data and materials The datasets used and analysed during the current study are available from the corresponding author on reasonable request.

\section{Compliance with ethical standards}

Conflict of interest The authors declare that they have no conflict of interest.

Open Access This article is licensed under a Creative Commons Attribution 4.0 International License, which permits use, sharing, adaptation, distribution and reproduction in any medium or format, as long as you give appropriate credit to the original author(s) and the source, provide a link to the Creative Commons licence, and indicate if changes were made. The images or other third party material in this article are included in the article's Creative Commons licence, unless indicated otherwise in a credit line to the material. If material is not included in the article's Creative Commons licence and your intended use is not permitted by statutory regulation or exceeds the permitted use, you will need to obtain permission directly from the copyright holder. To view a copy of this licence, visit http://creativecommons .org/licenses/by/4.0/.

\section{References}

1. Fédération Internationale de Football Association (2015) FIFA quality concept for football turf. www.FIFA.com. Accessed 23 Feb 2019

2. Fleming $P$ (2011) Artificial turf systems for sport surfaces: current knowledge and research needs. Proc Inst Mech Eng Part $P$ J Sports Eng Technol 225:43-63

3. Fleming P, Ferrandino $M$, Forrester $S$ (2016) Artificial turf field-a new build case study. Procedia Eng 147:836-841

4. Fédération Internationale de Football Association (2015) FIFA quality concept for football turf: handbook of requirements. www.FIFA.com. Accessed 23 Feb 2019 
5. McMahon TA, Greene PR (1979) The influence of track compliance on running. J Biomech 12(12):893-904

6. Petrass LA, Twomey DM (2013) The relationship between ground conditions and injury: what level of evidence do we have? J Sci Med Sport 16(2):105-112

7. Cole D, Forrester S, Fleming P (2018) Mechanical characterisation and modelling of elastomeric shockpads. Appl Sci 8(4):501

8. Bergström JS (2015) Mechanics of solid polymers: theory and computational modeling, 1st edn. William Andrew, pp 19-114

9. Ronkainen JA, El-Kati RF, Fleming PR, Forrester SE (2010) Application of an industrial robot in the sports domain: simulating the ground contact phase of running. Proc Inst Mech Eng Part P J Sports Eng Technol 224(4):259-269

10. Cavanagh PR (1987) The biomechanics of lower extremity action in distance running. Foot Ankle 7(4):197-217

11. Morin JB, Samozino P, Zameziati K, Belli A (2007) Effects of altered stride frequency and contact time on leg-spring behavior in human running. J Biomech 40(15):3341-3348

12. Burbank SD, Smith LV (2012) Dynamic characterization of rigid foam used in finite element sports ball simulations. Proc Inst Mech Eng Part P J Sports Eng Technol 226(2):77-85

13. Lane $B$, Sherratt $P, H u X$, Harland $A$ (2018) Measurement of strain and strain rate during the impact of tennis ball cores. Appl Sci 8(3):371

14. Fédération Internationale de Football Association (2015) FIFA quality concept for football turf: handbook of test methods. www.FIFA.com. Accessed 23 Feb 2019

15. Colino E, Sánchez-Sánchez J, García-Unanue J, Ubago-Guisado E, Haxaire P, Le Blan A, Gallardo L (2017) Validity and reliability of two standard test devices in assessing mechanical properties of different sport surfaces. Polym Test 62:61-67

16. Kolitzus HJ (2012) Scrutiny of advanced artificial athlete (AAA). In: ISSS technical conference Shanghai. www.isss-sportsurfa cescience.org $/$ page.. spp? node $=53 \&$ sec $=$ Conferences. Accessed 23 Feb 2019

17. Ramsden E (2006) Hall-effect sensors: theory and application, 2nd edn. Newnes, Burlington, pp 107-130

18. GOM mbH (2019) Photron product information. www.suppo rt.gom.com. Accessed 21 Mar 2019

19. Bland JM, Altman DG (1999) Measuring agreement in method comparison studies. Stat Methods Med Res 8(2):135-160

20. Burbank SD, Smith LV (2012) Dynamic characterization of rigid foam used in finite element sports ball simulations. Proc Inst Mech Eng Part P J Sports Eng Technol 226:77-85

21. Leach RJ, Forrester SE, Mears AC, Roberts JR (2017) How valid and accurate are measurements of golf impact parameters obtained using commercially available radar and stereoscopic optical launch monitors? Measurement 112:125-136

22. World Rugby (2016) Rugby turf performance specification. www. playerwelfare.worldrugby.org. Accessed 21 Mar 2019

Publisher's Note Springer Nature remains neutral with regard to jurisdictional claims in published maps and institutional affiliations. 